\title{
ÜBER MATRIZEN DIE BESCHRÄNKTE HALBGRUPPEN ERZEUGEN
}

\author{
HEINZ-OTTO KREISS
}

1. Einleitung. Wir wollen in dieser Arbeit folgendes Problem betrachten: Gegeben sei eine Familie $\mathfrak{F}$ von quadratischen Matrizen $A$ der Ordnung $n$ mit komplexen Elementen. Welche Bedingungen müssen die Matrizen $\in \mathfrak{F}$ erfüllen, damit $e^{A t}$ für alle $t \geqq 0$ und alle $A \in \mathfrak{F}$ gleichmässig beschränkt sind, d. h. eine Konstante $C_{1}$ existiert, so dass

$$
\left\|e^{A t}\right\| \leqq C_{1}
$$

ist, für alle $A \in \mathfrak{F}$ und alle $t \geqq 0$ ?

Dieses Problem hat eine sehr einfache Lösung, wenn die Matrizen $A$ normal sind. Bezeichnen wir nämlich mit $\varkappa_{i}$ die Eigenwerte der Matrizen $A$, so ist in diesem Fall

$$
\operatorname{Re} \varkappa_{i} \leqq 0
$$

die notwendige und hinreichende Bedingung dafür, dass (1.1) erfüllt ist. Es ist bekannt, dass diese Bedingung für Familien von nichtnormalen Matrizen zwar notwendig nicht aber hinreichend ist. Jedes notwendige und hinreichende Kriterium kann man daher als Mass für die Abweichung von der Normalität auffassen. Wir geben in dieser Arbeit drei solche Kriterien an. Wir beweisen nämlich den

Hauptsatz. Die folgenden Aussagen über eine Familie $\mathfrak{F}$ von Matrizen sind äquivalent:

1) Es gibt eine Konstante $C_{1}$, so dass für alle $A \in \mathfrak{F}$ und alle $t \geqq 0$

$$
\left\|e^{A t}\right\| \leqq C_{1} .
$$

2) Es gibt eine Konstante $C_{2}$, so dass für alle komplexen $s$ mit $\operatorname{Re} s>0$ und alle $A \in \mathfrak{F}$

$$
\left\|(A-I s)^{-\mathbf{1}}\right\| \leqq C_{2} / \operatorname{Re} s .
$$

Eingegangen am 1. April, 1959.

Diese Arbeit hat der Verfasser im Institut für Mathematik der Kgl. Technischen Hochschule geschrieben, während er Stipendiat des schwedischen technischen Forschungsrats war. 
3) Es gibt Konstanten $C_{31}$ und $C_{32}$ und $z u$ jeder Matrix $A \in \mathfrak{F}$ eine Matrix S, für welche

$$
\max \left(\|S\|,\left\|S^{-1}\right\|\right) \leqq C_{31}
$$

ist, so dass

$$
B=S A S^{-1}=\left(\begin{array}{ccccc}
\varkappa_{1} & b_{12} & \ldots & \ldots & b_{1 n} \\
0 & \varkappa_{2} & b_{23} & \ldots & b_{2 n} \\
\ldots & \ldots & \ldots & \ldots & \ldots \\
0 & \ldots & \ldots & 0 & \varkappa_{n}
\end{array}\right)
$$

ist, und die Ungleichungen

$$
\operatorname{Re} \varkappa_{i} \leqq \operatorname{Re} \varkappa_{j} \leqq 0, \quad j \leqq i,
$$

und

$$
\left|b_{i j}\right| \leqq C_{32}\left|\operatorname{Re} \varkappa_{i}\right|
$$

gelten.

4) Es existiert eine Konstante $C_{4}$ und zu jeder Matrix $A \in \mathfrak{F}$ eine positiv definite hermitische Matrix $H$, für welche

$$
\max \left(\|H\|,\left\|H^{-1}\right\|\right) \leqq C_{4}
$$

ist, so dass die Eigenwerte der normalen Matrix $H A+A^{*} H$ nicht positiv sind. (Mit $\|A\|$ wird die euklidische Norm einer Matrix $A$ und mit $A^{*}$ die zu $A$ adjungierte Matrix bezeichnet).

Den Beweis erbringen wir auf folgende Weise: Wir zeigen, dass sich aus jeder der obigen Aussagen die nächst folgende ergibt, und dass aus der letzten die erste folgt.

Die Aussagen dieses Satzes kann man mit Vorteil in der Theorie des ('auchyproblems für partielle Differential- und Differenzengleichungen anwenden. Vergleiche hierzu die Arbeiten des Verfassers [1] [2].

Professor Lars Hörmander machte mich auf die zweite Aussage des Hauptsatzes aufmerksam. Hierfür und für mehrere anregende Diskussionen möchte ich ihm danken.

2. Aus der ersten Aussage des Hauptsatzes folgt die zweite. Wir betrachten eine Familie F von Matrizen $A$, die die erste Aussage des Hauptsatzes erfüllt und wollen zeigen, dass für sie dann auch die zweite Aussage gilt. Bezeichnen wir mit $\varkappa_{i}$ die Eigenwerte der Matrizen $A$, so folgt aus (1.2) offensichtlich

$$
\operatorname{Re} x_{i} \leqq 0 .
$$

Daher existiert $(A-I s)^{-1}$ für $\operatorname{Re} s>0$, denn die Eigenwerte von $A-I s$ 
sind wegen (2.1) alle von 0 verschieden. Laplacetransformiert man $e^{A t}$, so ergibt sich aus (1.2)

$$
\begin{aligned}
\left\|(A-I s)^{-1}\right\|=\left|\int_{0}^{\infty} e^{(A-I s) t} d t\right| & =\int_{0}^{\infty} e^{-s t} e^{s t} d t \\
& \leqq C_{1} \int_{0}^{\infty}\left|e^{-s t}\right| d t=C_{1} / \operatorname{Re} s .
\end{aligned}
$$

3. Aus der zweiten Aussage der Hauptsatzes folgt die dritte. Wir betrachten zunächst Familien $\mathfrak{F}$ von Triangelmatrizen

$$
A=\left(\begin{array}{ccccc}
x_{1} & a_{12} & \ldots & \ldots & a_{1 n} \\
0 & x_{2} & a_{23} & \ldots & a_{2 n} \\
\ldots & \ldots & \ldots & \ldots & \ldots \\
0 & \ldots & \ldots & 0 & x_{n}
\end{array}\right)
$$

deren Eigenwerte auf folgende Weise angeordnet sind:

$$
\operatorname{Re} x_{i} \leqq \operatorname{Re} x_{j}, \quad j \leqq i .
$$

Für solche Familien können wir die in der Überschrift angegebene Behauptung in folgender verschärfter Form zeigen:

Satz 1. Gegeben sei eine Familie of von Triangelmatrizen der obigen Form, die die zweite Aussage des Hauptsatzes erfüllt. Dann gibt es Konstanten $C_{3 i}=C_{3 i}\left(C_{2}, n\right), i=1,2$, die nur von der Ordnung $n$ und der Konstanten $C_{2}$ abhängen, und zu jeder Matrix $A \in \widetilde{F}$ eine Matrix $S=S(A)$ der Form

$$
S=\left(\begin{array}{ccccc}
1 & s_{12} & \ldots & \ldots & s_{1 n} \\
0 & 1 & s_{23} & \ldots & s_{2 n} \\
\ldots & \ldots & \ldots & \ldots & \ldots \\
0 & \ldots & \ldots & 0 & 1
\end{array}\right) \quad \text { mit } \quad \max \left(\|S(A)\|,\left\|S^{-1}(A)\right\|\right) \leqq C_{31},
$$
so dass für

die L'ngleichungen

$$
B=S A S^{-1}=\left(\begin{array}{ccccc}
x_{1} & b_{12} & \ldots & \ldots & b_{1 n} \\
0 & x_{2} & b_{23} & \ldots & b_{2 n} \\
\ldots & \ldots & \ldots & \ldots & \ldots \\
0 & \ldots & \ldots & 0 & \varkappa_{n}
\end{array}\right)
$$

$$
\operatorname{Re} \varkappa_{i} \leqq \operatorname{Re} \varkappa_{j} \leqq 0, \quad j \leqq i,
$$

und

$$
\left|b_{i j}\right| \leqq C_{32}\left|\operatorname{Re} x_{i}\right|
$$

gelten. 
Beweis. (3.3) folgt offensichtlich aus (3.1) und (1.3). Die Aussagen (3.2) und (3.4) beweisen wir zunächst für $n=2$, um sie dann später durch vollständige Induktion für alle $n \mathrm{zu}$ beweisen. Für $\operatorname{Re} s>0$ gilt:

$$
(A-I s)^{-1}=\left(\begin{array}{cc}
\varkappa_{1}-s & a_{12} \\
0 & \varkappa_{2}-s
\end{array}\right)^{-1}=\left(\begin{array}{cc}
\left(\varkappa_{1}-s\right)^{-1}-a_{12}\left(\left(\varkappa_{1}-s\right)\left(\varkappa_{2}-s\right)\right)^{-1} \\
0 & \left(\varkappa_{2}-s\right)^{-1}
\end{array}\right) .
$$

Wegen (1.3) ist

$\mathrm{Da}$

$$
\left|a_{12}\right|\left|\left(\varkappa_{1}-s\right)\left(\varkappa_{2}-s\right)\right|^{-1} \leqq C_{2} / \operatorname{Re} s .
$$

so folgt

$$
\left|\varkappa_{1}-s\right| / \operatorname{Re} s \rightarrow 2 \quad \text { für } \quad s \rightarrow-\bar{\varkappa}_{1}+0,
$$

$$
\left|a_{12}\right| \leqq 2 C_{2}\left|\bar{\varkappa}_{1}+\varkappa_{2}\right|
$$

Setzt man

so ist

$$
S=\left(\begin{array}{cc}
1 & -\gamma \\
0 & 1
\end{array}\right) . \quad \gamma=\left\{\begin{array}{ccc}
0 & \text { für } & \bar{\varkappa}_{1}+\varkappa_{2}=0 \\
a_{12} /\left(\bar{\varkappa}_{1}+\varkappa_{2}\right) & \text { für } & \bar{\varkappa}_{1}+\varkappa_{2} \neq 0
\end{array},\right.
$$

$$
S^{-1}=\left(\begin{array}{ll}
1 & \gamma \\
0 & 1
\end{array}\right)
$$

und wir erhalten

$$
S A S^{-1}=\left(\begin{array}{ccc}
\varkappa_{1} & 2 \gamma & \operatorname{Re} \varkappa_{1} \\
0 & \varkappa_{2}
\end{array}\right) .
$$

Da nach (3.5) die Ungleichung $|\gamma| \leqq 2 C_{2}$ gilt, so haben wir die Aussagen (3.2) und (3.4) für $n=2$ bewiesen.

Auch für $n=1$ sind die Aussagen (3.2) und (3.4) offensichtlich richtig. Seien sie jetzt für die Ordnung $v=n-1, v \geqq 2$ bewiesen. Wir zeigen, dass sie dann auch für $\nu=n$ gelten. Dazu betrachten wir eine feste Matrix $A \in \mathfrak{F}$. Diese kann man in der Form

$$
A=\left(\begin{array}{cc}
A_{1} & a_{1} \\
0 & x_{n}
\end{array}\right)
$$

schreiben, wobei

$$
A_{1}=\left(\begin{array}{ccccc}
\varkappa_{1} & a_{12} & \ldots & \ldots & a_{1 n-1} \\
0 & \varkappa_{2} & a_{23} & \ldots & a_{2 n-1} \\
\ldots & \ldots & \ldots & \ldots & \ldots \\
0 & \ldots & \ldots & 0 & x_{n-1}
\end{array}\right), \quad a_{1}=\left(\begin{array}{c}
a_{1 n} \\
\vdots \\
\vdots \\
a_{n-1 n}
\end{array}\right)
$$

sind. Da $(A-I s)^{-1}$ von der Form

$$
\left(\begin{array}{cc}
\left(A_{1}-I_{1} s\right)^{-1} & \hat{a}_{1} \\
0 & \left(\varkappa_{n}-s\right)^{-1}
\end{array}\right)
$$

ist, gilt (1.3) auch für $A_{1}$. Also existieren nach Induktionsvoraussetzung 
Konstanten $C_{3 i}{ }^{\prime}=C_{3 i}\left(C_{2}, n-1\right)$ und eine Matrix $S=S\left(A_{1}\right)$ der obigen Art, so dass für $S A_{1} S^{-1}$ die Aussagen des Satzes erfüllt sind. Daher gilt:

$$
\begin{gathered}
\left(\begin{array}{ll}
S & 0 \\
0 & 1
\end{array}\right)\left(\begin{array}{cc}
A_{1} & a_{1} \\
0 & x_{n}
\end{array}\right)\left(\begin{array}{cc}
S^{-1} & 0 \\
0 & 1
\end{array}\right)=\left(\begin{array}{cc}
S A_{1} S^{-1} & S a_{1} \\
0 & x_{n}
\end{array}\right)=A^{\prime} \\
=\left(\begin{array}{ccccc}
x_{1} & a_{12} & \ldots & \ldots & a_{1 n}^{\prime} \\
0 & \varkappa_{2} & a_{23}^{\prime} & \ldots & a_{2 n}{ }^{\prime} \\
\ldots & \ldots & \ldots & \ldots & \ldots \\
0 & \ldots & \ldots & \ldots & \varkappa_{n}
\end{array}\right),
\end{gathered}
$$

und alle $a_{i j}{ }^{\prime}$ mit $j<n$ erfüllen die Aussage (3.4) des Satzes mit $C_{32}=C_{32}{ }^{\prime}$. Setzt man

so folgt für $\operatorname{Re} s>0$

$$
S_{1}=\left(\begin{array}{ll}
S & 0 \\
0 & 1
\end{array}\right)
$$

$$
\begin{aligned}
\left\|\left(A^{\prime}-I s\right)^{-1}\right\| & =\left\|\left(S_{1} A S_{1}^{-1}-I s\right)^{-1}\right\| \\
& \leqq\left\|S_{1}{ }^{-1}\right\|\left\|S_{1}\right\|\left\|(A-I s)^{-1}\right\| \leqq C_{2}{ }^{\prime} / \operatorname{Re} s,
\end{aligned}
$$

wobei $C_{2}{ }^{\prime}=C_{2} \cdot C_{31}{ }^{\prime 2}$ nur von $C_{2}$ und $n-1$ abhängt.

Die Matrix $A^{\prime}$ kann man in der Form

schreiben, wobei

$$
A=\left(\begin{array}{cc}
x_{1} & a_{2}^{\prime} \\
0 & A_{2}^{\prime}
\end{array}\right)
$$

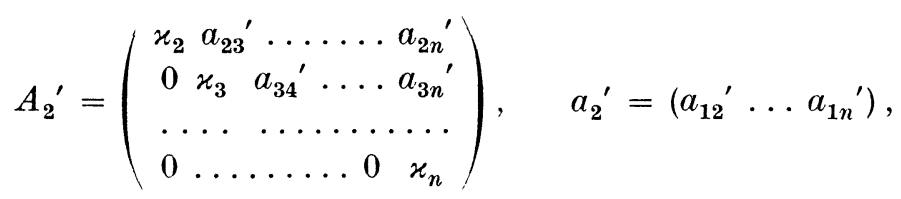

sind. Genau so wie oben folgt, dass die Abschätzung (3.6) auch für $A_{2}^{\prime}$ gilt. Also gibt es nach Induktionsvoraussetzung Konstanten $C_{3 i}{ }^{\prime \prime}=$ $C_{3 i}\left(C_{2}{ }^{\prime}, n-1\right)$ und eine Matrix $S^{\prime}=S^{\prime}\left(A_{2}{ }^{\prime}\right)$, so dass für $S^{\prime} A_{2}{ }^{\prime} S^{\prime-1}$ die Aussagen des Satzes erfüllt sind. Daher gilt

$$
\begin{aligned}
& \left(\begin{array}{cc}
1 & 0 \\
0 & S^{\prime}
\end{array}\right)\left(\begin{array}{cc}
\varkappa_{1} & a_{2}{ }^{\prime} \\
0 & A_{2}^{\prime}
\end{array}\right)\left(\begin{array}{cc}
1 & 0 \\
0 & S^{\prime-1}
\end{array}\right)=\left(\begin{array}{cc}
\varkappa_{1} & a_{2}{ }^{\prime} S^{\prime-1} \\
0 & S^{\prime} A_{2}{ }^{\prime} S^{\prime-1}
\end{array}\right)=A^{\prime \prime} \\
& =\left(\begin{array}{ccccc}
x_{1} & a_{12}{ }^{\prime \prime} & \ldots & \ldots & a_{1 n}{ }^{\prime \prime} \\
0 & x_{2} & a_{23}{ }^{\prime \prime} & \ldots & a_{2 n}{ }^{\prime \prime} \\
\ldots & \ldots & \ldots & \ldots & \ldots \\
0 & \ldots & \ldots & 0 & x_{n}
\end{array}\right),
\end{aligned}
$$

und alle $a_{i j}{ }^{\prime \prime}$ mit $i>1$ erfüllen die Aussage (3.4) mit $C_{32}=C_{32}{ }^{\prime \prime}$. Da ausserdem $a_{1 j}{ }^{\prime \prime}$ nur von den $a_{1 i}{ }^{\prime \prime}$ (und zwar linear) abhängen, für die 
$i \leqq j$ ist, so gibt es eine Konstante $C_{32}{ }^{\prime \prime \prime}$, die nur von $C_{2}{ }^{\prime}$ und $n-1, \mathrm{~d} . \mathrm{h}$. nur von $C_{2}$ und $n-1$ abhängt, so dass alle $a_{i j}{ }^{\prime \prime}$ ausser $a_{1 n}{ }^{\prime \prime}$ die Aussage (3.4) mit $C_{32}=C_{32}{ }^{\prime \prime \prime}$ erfüllen. Aus (3.6) folgt, wenn man statt $S_{1}$ die Matrix

$$
S_{2}=\left(\begin{array}{cc}
1 & 0 \\
0 & S^{\prime}
\end{array}\right)
$$

einsetzt:

$$
\left\|\left(A^{\prime \prime}-I s\right)^{-1}\right\| \leqq C_{2}{ }^{\prime \prime} / \operatorname{Re} s, \quad C_{2}{ }^{\prime \prime}=C_{2}{ }^{\prime} \cdot C_{31}{ }^{\prime \prime 2} .
$$

Betrachten wir jetzt das $n$-te Element $c_{1 n}$ in der ersten Zeile von $\left(A^{\prime \prime}-I s\right)^{-1}$. Nach Cramers Regel gilt:

mit

$$
\begin{aligned}
c_{1 n} & =(-1)^{n-1} \prod_{i=1}^{n}\left(\varkappa_{i}-s\right)^{-1} \cdot D_{1} \\
& =\frac{-a_{1 n}{ }^{\prime \prime}}{\left(\varkappa_{1}-s\right)\left(\varkappa_{n}-s\right)}+\frac{(-1)^{n-1}}{\left(\varkappa_{n}-s\right)} \cdot D_{2},
\end{aligned}
$$

und

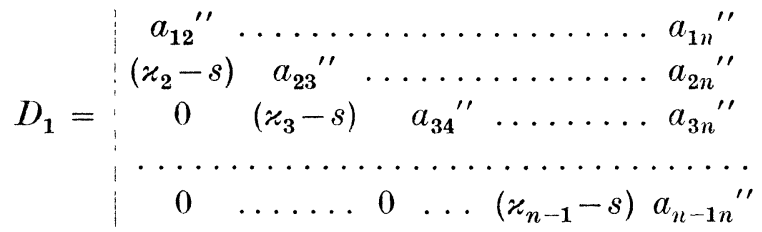

$$
D_{2}=\left|\begin{array}{ccccccc}
g_{12} & \ldots & \ldots & g_{1 n-1} & 0 \\
1 & g_{23} & \ldots & \ldots & \ldots & g_{2 n} \\
0 & 1 & g_{34} & \ldots & \ldots & g_{3 n} \\
\ldots & \ldots & \ldots & \ldots & \ldots & \ldots \\
0 & \ldots & \ldots & 0 & 1 & g_{n-1 n}
\end{array}\right|
$$

und wobei $g_{i j}=a_{i j}{ }^{\prime \prime} /\left(\varkappa_{i}-s\right)$ ist. Da $a_{1 n}{ }^{\prime \prime}$ in der Determinante $D_{2}$ nicht vorkommt und alle anderen $a_{i j}{ }^{\prime \prime}$ die Aussage (3.4) mit $C_{32}=C_{32}{ }^{\prime \prime \prime}$ erfüllen und für die $\varkappa_{i}$ die Ungleichungen (3.3) gelten, so existiert eine Konstante $C_{2}{ }^{\prime \prime \prime}$, die nur von $C_{2}$ und $n-1$ abhängt, so dass für $\operatorname{Re} s>0$

$$
\frac{(-1)^{n-1}}{x_{n}-s} \cdot D_{2} \mid \leqq \frac{C_{2}{ }^{\prime \prime \prime}}{\left|\varkappa_{n}-s\right|} \leqq \frac{C_{2}{ }^{\prime \prime \prime}}{\operatorname{Re} s}
$$

ist. Wegen (3.7) gilt daher

$$
\left|a_{1 n}{ }^{\prime \prime}\right| /\left|\left(\varkappa_{1}-s\right)\left(\varkappa_{n}-s\right)\right| \leqq\left(C_{2}{ }^{\prime \prime}+C_{2}{ }^{\prime \prime \prime}\right) / \operatorname{Re} s=C_{2}{ }^{1 \mathrm{v}} / \operatorname{Re} s,
$$

und $C_{2}$ IV hängt nur von $C_{2}$ und $n-1$ ab. Daraus folgt genauso wie (3.5)

$$
\left|a_{1 n}^{\prime \prime}\right| \leqq 2 C_{2}^{\mathrm{IV}}\left|\bar{x}_{1}+\varkappa_{n}\right| \text {. }
$$


Setzen wir jetzt

$$
S_{3}=I-\gamma \cdot\left(\begin{array}{cccc}
0 & \ldots & 0 & 1 \\
0 & \ldots & 0 & 0 \\
\ldots & \ldots & \ldots \\
0 & \ldots & 0
\end{array}\right), \quad \gamma=\left\{\begin{array}{ccc}
0 & \text { für } & \bar{\varkappa}_{1}+\varkappa_{n}=0 \\
a_{1 n}{ }^{\prime \prime} /\left(\bar{\varkappa}_{1}+\varkappa_{n}\right) & \text { für } & \bar{\varkappa}_{1}+\varkappa_{n} \neq 0
\end{array},\right.
$$

so ist

$$
S_{3}^{-1}=I+\gamma\left(\begin{array}{cccc}
0 & \ldots & 0 & 1 \\
0 & \ldots & 0 & 0 \\
\ldots & \ldots & 0 \\
0 & \ldots & 0 & 0
\end{array}\right)
$$

und

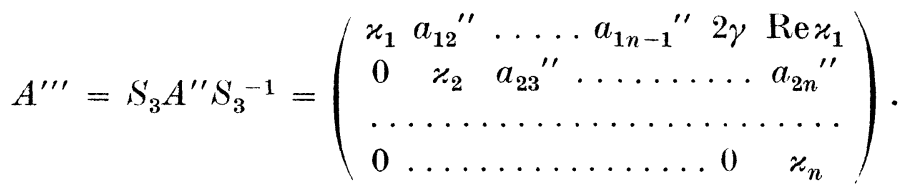

Wegen (3.8) folgt daraus aber der Satz 1. Denn wir haben zu einer beliebigen Matrix $A \in \mathfrak{F}$ induktiv Konstanten $C_{31}\left(C_{2}, n\right), C_{32}\left(C_{2}, n\right)$ bestimmt, die nicht von $A$ sondern nur von $C_{2}$ und $n$ abhängen, und eine Matrix $S(A)=S_{3} S_{2} S_{1}$ konstruiert, so dass die Aussagen (3.2) und (3.4) des Satzes mit $C_{3 i}\left(C_{2}, n\right)$ erfüllt sind.

Man beachte besonders, dass die Beweismethode gleichzeitig auch eine Möglichkeit ergibt, die Normalform $S A S^{-1}$ für jedes $A$ in endlich vielen Schritten zu konstruieren.

Die Voraussetzung des Satzes 1, dass $\mathfrak{F}$ eine Familie von Triangelmatrizen ist, für die (3.1) gilt, ist nicht wesentlich, da man jede Matrix $A$ mit Hilfe einer unitären Transformation auf Triangelgestalt mit der Eigenschaft (3.1) transformieren kann. Da durch eine solche Transformation die Norm von $A-I s$ nicht geändert wird, so gilt Satz 1 für beliebige Matrizen, wenn man als Transformation $S(A)$ Transformationen der Form

$$
S(A)=\left(\begin{array}{ccccc}
1 & s_{12} & \ldots & \ldots & s_{1 n} \\
0 & 1 & s_{23} & \ldots & s_{2 n} \\
\ldots & \ldots & \ldots & \ldots & \ldots \\
0 & \ldots & \ldots & 0 & 1
\end{array}\right) \cdot U
$$

zulässt, wobei $U$ geeignete unitäre Transformationen sind. Damit haben wir gezeigt, dass für jede Familie $\mathfrak{F}$ von Matrizen $A$, die die zweite Aussage des Hauptsatzes erfüllt, auch die dritte Aussage gilt. 
4. Aus der dritten Aussage des Hauptsatzes folgt die vierte. Wir betrachten zunächst wieder Familien von Triangelmatrizen und beweisen den

Satz 2. Gegeben sei eine Konstante $C \geqq 0$. Es sei $\mathfrak{f}$ die Familie aller Triangelmatrizen

$$
A=\left(\begin{array}{ccccc}
x_{1} & a_{12} & \ldots & \ldots & a_{1 n} \\
0 & x_{2} & a_{23} & \ldots & a_{2 n} \\
\ldots & \ldots & \ldots & \ldots & \ldots \\
0 & \ldots & \ldots & 0 & x_{n}
\end{array}\right),
$$

für die die Ungleichungen

$$
\operatorname{Re} \varkappa_{i} \leqq \operatorname{Re} \varkappa_{j} \leqq 0, \quad j \leqq i,
$$

und

$$
\left|a_{i j}\right| \leqq C\left|\operatorname{Re} \varkappa_{i}\right|
$$

gelten. Dann existiert eine positive Diagonalmatrix

$$
D=\left(\begin{array}{ccccc}
d & 0 & \ldots & \ldots & 0 \\
0 & d^{2} & 0 & \ldots & 0 \\
\ldots & \ldots & \ldots & 0 \\
0 & \ldots & \ldots & 0 & d^{n}
\end{array}\right), \quad d>1
$$

so dass für jedes $A \in \mathfrak{F}$ die Eigenwerte von $D A+A^{*} D$ kleiner oder gleich Null sind.

Beweis. Die Eigenwerte von $D A+A^{*} D$ sind die Lösungen der algebraischen Gleichung:

$$
\operatorname{Det}\left(D A+A^{*} D-I \varkappa\right)=d^{\frac{1}{2} n(n+1)} \cdot \prod_{i=1}^{n}\left(\left(\varkappa_{i}+\bar{\varkappa}_{i}\right)-\varkappa d^{-i}\right) \cdot \operatorname{Det}\left(g_{i j}\right)=0,
$$

wobei

$$
g_{i j}=\left\{\begin{array}{lll}
a_{i j}\left(\left(\varkappa_{i}+\bar{\varkappa}_{i}\right)-x d^{-i}\right)^{-1} & \text { für } \quad i<j \\
1 & \text { für } i=j \\
\bar{a}_{j i}\left(\left(\varkappa_{i}+\bar{x}_{i}\right)-x d^{-i}\right)^{-1} d^{-(i-j)} & \text { für } i>j .
\end{array}\right.
$$

Wegen (4.1) und (4.2) folgt daher für alle $\varkappa>0$ und alle $A \in \mathfrak{F}$

$$
\left|g_{i j}\right| \leqq\left\{\begin{array}{cl}
C & \text { für } \quad i<j \\
C \cdot d^{-(i-j)} & \text { für } \quad i>j .
\end{array}\right.
$$

Also gilt gleichmässig für alle $\varkappa>0$ und alle $A \in \mathfrak{F}$

$$
\operatorname{Det}\left(g_{i j}\right) \rightarrow 1 \quad \text { wenn } \quad d \rightarrow \infty,
$$

und daraus ergibt sich die Behauptung unmittelbar. 
Es sei jetzt $\mathfrak{F}$ eine Familie von Matrizen $A$, die die dritte Aussage des Hauptsatzes erfüllt. Wir wollen zeigen, dass für sie auch die vierte Aussage gilt. Nach Satz 2 gibt es eine positive Diagonalmatrix (4.3), deren Elemente durch eine Konstante beschränkt sind, die nur von $C_{32}$ abhängt, so dass die Eigenwerte von

$$
D S A S^{-1}+S^{*-1} A^{*} S^{*} D
$$

kleiner oder gleich Null sind. Also ist die quadratische Form

$$
u^{*}\left\{D S A S^{-1}+S^{*-1} A^{*} S^{*} D\right\} u, \quad u^{*}=\left(u_{1}, \ldots, u_{n}\right)
$$

negativ semidefinit. Setzt man $v=S^{-1} u$, so kann man (4.5) auch als Form für $v$ schreiben

$$
v^{*}\left\{S^{*} D S A+A * S * D S\right\} v .
$$

Daher sind die Eigenwerte von $S^{*} D S A+A^{*} S^{*} D S$ nicht positiv und die gesuchte Matrix $H$ ist

$$
H=S^{*} D S
$$

5. Aus der vierten Aussage des Hauptsatzes folgt die erste. Wir betrachten eine Familie $\mathfrak{F}$ von Matrizen $A$, die die vierte Aussage des Hauptsatzes erfüllt. Wir wollen zeigen, dass für sie dann auch die erste Aussage gilt. Zur Abkürzung setzen wir $e^{A t}=F(t)$ und betrachten die quadratische Form

Es gilt

$$
u^{*} F^{*}(t) H F(t) u \text {. }
$$

$$
\frac{d}{d t}\left(u^{*} F^{*}(t) H F(t) u\right)=u^{*} F^{*}(t)\left(H A+A^{*} H\right) F(t) u \leqq 0 .
$$

Also ist für alle $t \geqq 0$

$$
u^{*} F^{*}(t) H F(t) u \leqq u^{*} H u .
$$

Nun ist für alle $v$

$$
C_{4}^{-1} v^{*} v \leqq v^{*} H v \leqq C_{4} v^{*} v .
$$

Aus (5.1) folgt dann

Also ist

$$
C_{4}^{-1}\|F(t) u\|^{2} \leqq u^{*} F^{*}(t) H F(t) u \leqq u^{*} H u \leqq C_{4}\|u\|^{2} .
$$

$$
\left\|e^{A t}\right\| \leqq C_{4} .
$$

Damit haben wir aber den Hauptsatz vollständig bewiesen. 


\section{LITERATUR}

1. H.-O. Kreiss, Uber sachgemässe Cauchyprobleme für Systeme von linearen partiellen Differentialgleichungen, Trans. Roy. Inst. Technology, Stockholm, Nr. 127 (1958).

2. H.-O. Kreiss, Uber die approximative Lösung von linearen partiellen Differentialgleichungen mit Hilfe von Differenzengleichungen, Trans. Roy. Inst. Technology, Stockholm, Nr. 128 (1958).

KGL. TECHNISCHE HOCHSCHLLE, STOCKHOLM. SCHWEDEN 